\title{
Nonlinear and tunable metamaterials
}

\author{
Yuri S. Kivshar \\ Nonlinear Physics Center, Research School of Physics and Engineering, \\ Australian National University, Canberra ACT 0200, Australia
}

\begin{abstract}
We review the recent experimental results from our Nonlinear Physics Center on tunability and nonlinear response of microstructured metamaterials with negative refractive index. We suggest and design new types of tunable metamaterials exhibiting either nonlinear magnetic or nonlinear electric response at microwave frequencies. By introducing a varactor diode as a nonlinear element within each resonator, we shift the frequency of either magnetic or electric resonance by changing the incident power. We also discuss a novel approach for efficient tuning of the transmission characteristics of metamaterials through a continuous adjustment of the lattice structure, the so-called structural tunability. Some of the tuning mechanisms discussed here can be suitable for scaling toward optical wavelengths with useful applications realizable in a wide frequency range.
\end{abstract}

Keywords: Nonlinear metamaterials, tunability, frequency shift, nonlinear resonance, structural tunability

\section{INTRODUCTION}

Both theoretical predictions ${ }^{1-3}$ and experimental fabrication and measurements ${ }^{4,5}$ have proven a possibility for creating novel types of microstructured materials that demonstrate many unique properties not found in Nature, including the negative index of refraction. One of the first realizations of such composite materials was created by a lattice of wires and split-ring resonators, and it was shown to possess a negative real part of magnetic permeability and dielectric permittivity for microwaves. Such metamaterials are also referred to as left-handed materials or materials with negative refraction. Properties of such materials were analyzed theoretically by Veselago a long time ago, ${ }^{6}$ but they became a subject of active experimental research only recently.

Metamaterials are prominent for exceptional opportunities they offer in tailoring macroscopic properties of materials through appropriate choice and arrangement of their structural elements. ${ }^{7,8}$ In this way, it is not only possible to design a metamaterial for a required functionality, but also to implement further adjustment capabilities at the level of assembly. This makes metamaterials different from conventional materials and opens exciting opportunities to implement tunability.

Tunable metamaterials imply the ability to continuously change their properties through certain external influence or signal, with the mechanism of tunability being intrinsic to metamaterial. The key means of tuning resonant metamaterials, naturally, lies in affecting the system so as to change the parameters of the resonance. As a consequence, characteristics of metamaterial can be varied, enabling tunable transmission.

Generally speaking, there are several means to achieve tunability of metamaterials. This includes

1. Modification of a basic element of the composite structure, e.g. a split-ring resonator or a cut-wire pair. For microwaves, this is achieved by introducing a varactor diode as externally or nonlinearly tunable element. This leads to a shift of the split-ring resonator frequency, and for a composite structure, to either magnetic or electric (or both) response by changing the incident power. ${ }^{9-12}$

2. Selection of a tunable substrate supporting the layers of elements, such as split-ring resonators; one can employ the specific properties of the substrate to achieve the tunable response of the whole structure. ${ }^{13-15}$ Similarly, the substrate may change its properties under external illumination

3. Control of metamaterial properties through engineering the structure geometry which modifies near-field coupling between the elements, the so-called structural tunability ${ }^{16,17}$

E-mail: ysk124@rsphysse.anu.edu.au,FAX: $+61-26-125-8588$

Metamaterials: Fundamentals and Applications II, edited by Mikhail A. Noginov, Nikolay I. Zheludev, Allan D. Boardman, Nader Engheta, Proc. of SPIE Vol. 7392, 739217 - @ 2009 SPIE · CCC code: 0277-786X/09/\$18 - doi: 10.1117/12.827872 
The possibility to control the effective parameters of a metamaterial using nonlinear response of the split-ring resonators has been suggested some time ago, ${ }^{18-20}$ but only recently these ideas have been implemented in a novel type of nonlinear tunable structures. ${ }^{21,22}$ The similar approach can be employed for creating nonlinear electric metamaterials. ${ }^{23}$ However, such methods become increasingly difficult to implement at higher frequencies. Some other approaches developed very recently employ an analogy with natural materials which demonstrate different properties depending on their specific crystalline structures. ${ }^{17}$ Metamaterials allow realizing a great opportunity for a design of the unique structures with a desired response function and implementing convenient mechanism for tunability. More importantly, the range of tunability for a given property can be much larger than in natural materials, as the lattice effects can be made much stronger through higher efficiency of collective effects in the lattice, achieved by an appropriate design.

In this paper we present a brief review of the recent results from our Nonlinear Physics Center on tunable and nonlinear metamaterials, as well as discuss new types of tunable structures exhibiting either nonlinear magnetic or nonlinear electric response at microwave frequencies. We also discuss a novel approach for efficient tuning of the transmission characteristics of metamaterials through a continuous adjustment of the lattice structure, structural tunability. Some of the tuning mechanisms discussed here can be suitable for scaling toward optical wavelengths and useful applications realizable in a wide frequency range.

The paper is organized as follows. In Sec. 2 we discuss tunability of a single split-ring resonator as a basic element for creating tunable and nonlinear metamaterials. We introduce a varactor diode as a nonlinear tunable element into each resonator, and shift the frequency of either magnetic or electric resonance by changing either external bias or incident power. Nonlinear magnetic and electric metamaterials based on such tunable split-ring resonators are discussed in Sec. 3 and Sec. 4, respectively. Then, in Sec. 5 we discuss a different approach for efficient tuning of metamaterials based on a change of the lattice structure and modification of the properties originated from the near-field interaction between the neighboring elements. Finally, Sec. 6 concludes the paper.

\section{TUNABILITY OF SINGLE SPLIT-RING RESONATORS}

Effective control over the resonant conditions of the split-ring resonator is achieved by adding the capacitance of the diode in series with the distributed capacitance of the outer ring of the resonator at a point of maxima in the electric currents. The series application of the diode provides a simple mechanism for both tunability and nonlinearity suited to the formation of metamaterials, particularly with the recent developments in magnetic thin-film and microwave nonlinear materials. The symmetry and simplicity of such systems also lends itself to greater integration, allowing the structure to be translated more readily to the $\mathrm{THz}$ and optical frequency domains.

To study both tunability and nonlinearity of metamaterials, we use a single archetype split-ring resonator constructed on fibreglass (FR4, $\epsilon_{r} \approx 4.4$ ) with copper metallization, ${ }^{9}$ as shown in Fig. 1(left, a). We take a resonator with an internal ring radius of $2.56 \mathrm{~mm}$. Both rings have a linear width of $1.44 \mathrm{~mm}$, and the separation between rings as well as the ring slots are $0.32 \mathrm{~mm}$. The simple biasing circuit consist of wire-wound shunt inductors (L1, L2) and de-coupling capacitor (C1), as illustrated in the schematic of Fig. 1(left, b). For a negative bias voltage of -10 volts (not shown) the resonant frequency can be shifted to $2.9 \mathrm{GHz}$, whereas for a positive voltage of 1 volt the resonant frequency decreases to $2.27 \mathrm{GHz}$. For this particular varicap diode and split-ring resonator structure there is a tuning bandwidth of $0.63 \mathrm{GHz}$, equivalent to a tuning range of approximately $26 \%$.

Different tuning regimes can be achieved by adding an inductive coil in parallel with the varactor. ${ }^{10}$ The coil can change the sign of the nonlinearity and eliminates the memory effect caused by charge accumulation across the varactor. In addition, at higher powers the nonlinear response of the split-ring resonator becomes multi-valued, paving a way for creating bistable tunable metamaterials. ${ }^{10}$

The varactor diode introduced in the split-ring resonator alters the resonant frequency $\omega_{r}$,

$$
\omega_{r}=\omega_{0}\left(1+\frac{C_{\mathrm{SRR}}}{C_{\mathrm{eff}}}\right)^{1 / 2} .
$$

Proc. of SPIE Vol. 7392 739217-2 

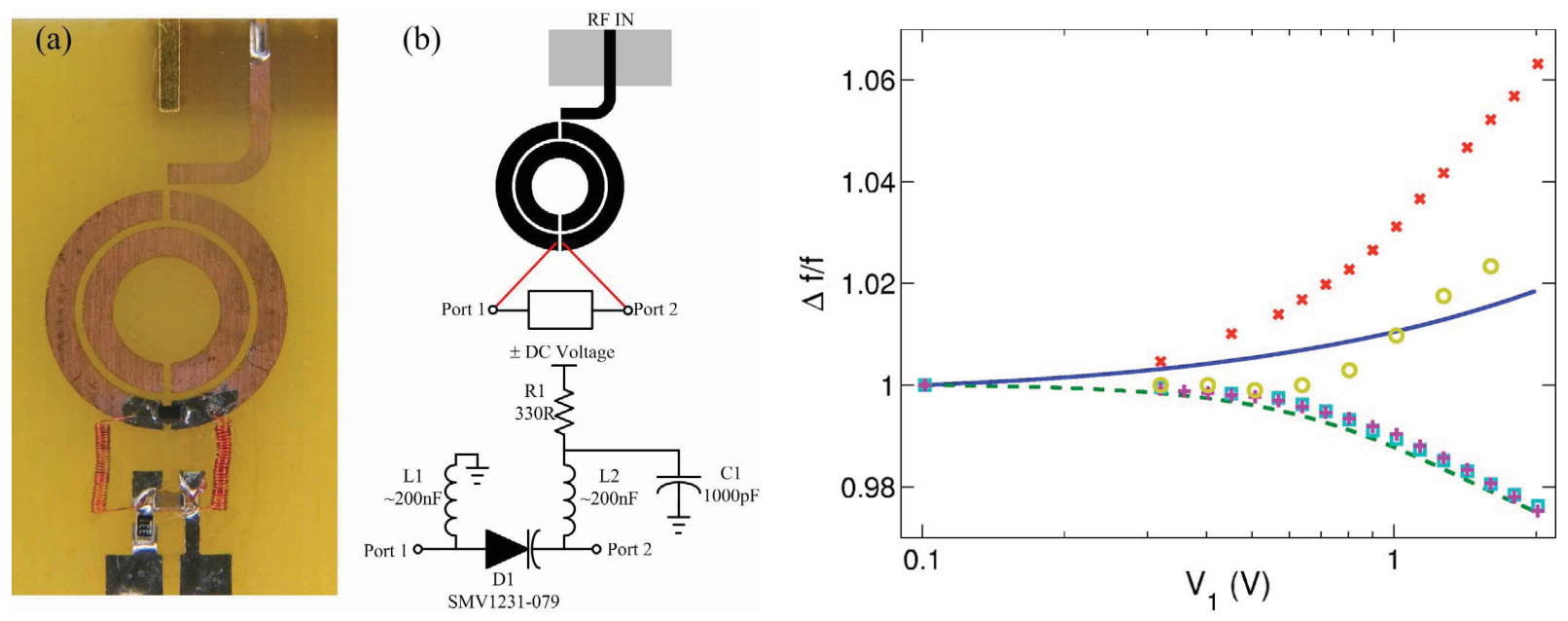

Figure 1. Left: Experimental structure used to study tunability and nonlinearity of a varactor-loaded split-ring resonator system. (a) Photography showing the fabricated split-ring resonator and biasing circuitry used for direct voltage tuning of the resonant frequency. (b) Top: electromagnetic representation of the resonator-diode structure; bottom: schematic of the biasing circuit. Right: Shift of the resonant frequency of the split-ring resonator vs diode rf voltage from Eq. (1) without (solid line) and with (dashed line) coil, calculated numerically without (circles) and with (straight crosses) coil, and measured without (tilted crosses) and with (squares) coil.

Here the notations $C_{\mathrm{eff}}$ and $C_{\mathrm{SRR}}$ stand for the capacitances of a varactor diode and split-ring resonator, respectively, and $\omega_{0}=\left(L_{\mathrm{SRR}} C_{\mathrm{SRR}}\right)^{1 / 2}$, where the index "SRR" refer to the split-ring resonator.

We have found that the diode discharges very slowly without the coil. Thus it was necessary to gradually scan through the frequency range to ensure that the measurements at each frequency were not affected by the rectification occurring at another. The resonant frequency was determined from the minimum of the reflection coefficient, and its shift is presented in Fig. 1(right) for the cases with and without a coil. The theoretical results obtained with fitted values of capacitance from Eq. (1) are also plotted.

The configuration without the coil shows stronger nonlinearity, but it has a pronounced memory effect, which slows down the response to changes in the input power. Additionally, the resonator without the coil was found to be highly sensitive to interference from electrical wiring within the building. ${ }^{9}$ This problem is largely eliminated once the resonator is placed inside the cavity. These results pave a way for creating nonlinear active metamaterials composed of tunable split-ring resonators, as discussed in two sections below.

\section{NONLINEAR MAGNETIC METAMATERIALS}

Nonlinear magnetic metamaterials operating at microwave frequencies can be fabricated by modifying the properties of split-ring resonators and introducing varactor diodes in each element of the composite structure, ${ }^{9,10}$ such that the whole structure becomes dynamically tunable by varying the amplitude of the propagating electromagnetic waves. In this way, we can demonstrate the power-dependent transmission of the magnetic metamaterials at higher powers, ${ }^{21,22}$ as was suggested earlier theoretically, ${ }^{18}$ and realize experimentally the nonlinearitydependent enhancement or suppression of the transmission in dynamically tunable magnetic metamaterial.

Metamaterial sample [see Fig. 2(left)] is fabricated from $0.5 \mathrm{~mm}$ thick Rogers R4003 printed circuit boards with nominal dielectric constant of 3.4. We make dielectric boards with the appropriate slot allocations with tin coated copper nonlinear split-ring resonators. Photograph of one of several nonlinear metamaterial structures is shown in Fig. 2(left). Each split-ring resonator contains variable capacity diode (model Skyworks SMV-1405) which introduces nonlinear current-voltage dependence and results in nonlinear magnetic dipole moment to each split-ring resonator. ${ }^{9}$ In terms of effective medium parameters, the manufactured structure has nonlinear 

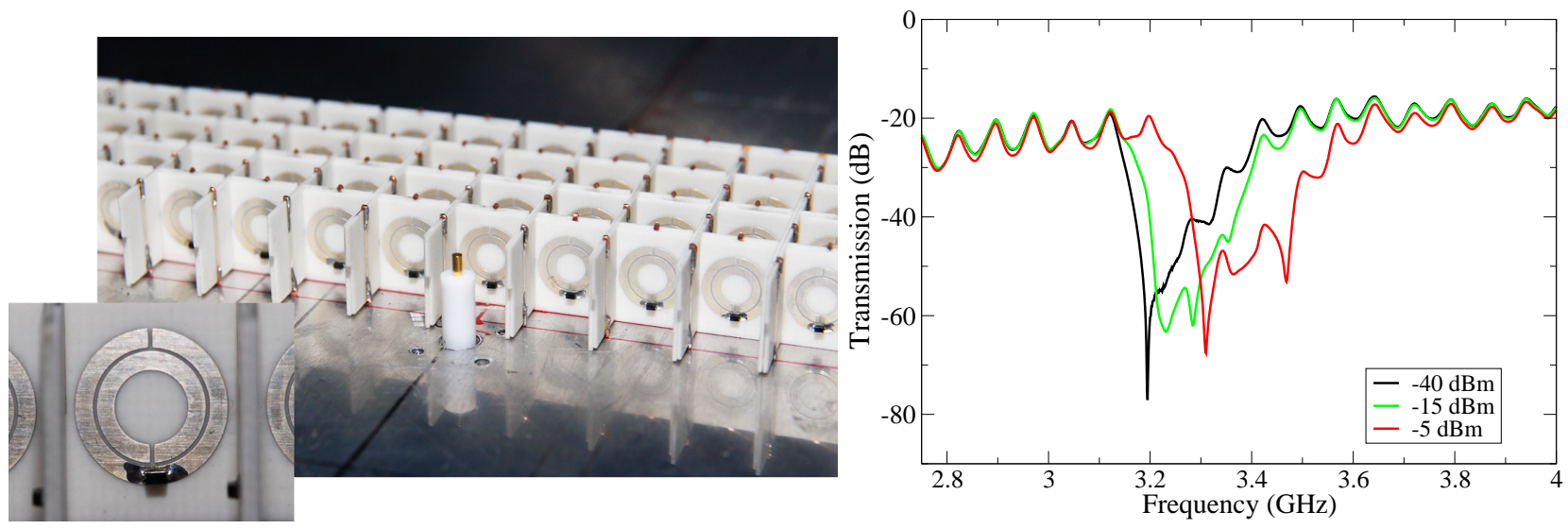

Figure 2. Left: Photograph of the nonlinear tunable magnetic metamaterial created by a square lattice of nonlinear splitring resonators. Each split-ring resonator contains a varactor which provides the power-dependant nonlinear response . Right: Measured transmission coefficients of the nonlinear magnetic metamaterial at different power levels indicated on the plot.

magnetization and nonlinear effective magnetic permittivity. ${ }^{18}$ Arrays of split-ring resonators form a twodimensional square lattice with $29 \times 4 \times 1$ unit cells of the size of $10.5 \mathrm{~mm}$.

First, to identify the effect of the nonlinearity we measure the transmission properties of the tunable magnetic metamaterial for different values of the input power. To measure the electromagnetic field scattering for our samples, the metamaterial slab is placed in a parallel plate waveguide. The planes of split-ring resonators are aligned perpendicular to the parallel plate surfaces. The input antenna is placed at the midpoint of the lower plate, $2 \mathrm{~mm}$ from the metamaterial slab, in front of the central unit cell, and it consists of a teflon-coated conductor of $1.26 \mathrm{~mm}$ diameter and $11 \mathrm{~mm}$ long. The teflon coating provides a better energy coupling into the waveguide for the wavelengths of interest. The antenna is positioned perpendicular to the bottom plate, so that the excited electric field is polarized perpendicular to the plane, and thus parallel to the wires. The magnetic field of the wave has mainly an in-plane component, effectively exciting the split-ring resonators. Close positioning of the source antenna to the metamaterial was chosen in order to funnel high EM power into the metamaterial sample in order to observe nonlinear effects. We note that different positioning of the source antenna with respect to the central unit cell of the metamaterial gives slightly different quantitative results for the measured transmission, however qualitatively all the results are identical. This effect appears due to different antenna impedance matching to the sample. An identical antenna is placed in the center of the top plate, and is used as receiver for spectra measurements and for raster scan of the electric field distribution in the horizontal plane. The input antenna is excited using an Agilent E8364A vector network analyzer, which output is amplified by HP $83020 \mathrm{~A} 38 \mathrm{~dB}$ amplifier. For the transmission measurements, the receiving antenna is located $2 \mathrm{~cm}$ behind the metamaterial slab, in front of the central unit cell of the metamaterial, and it is connected to the network analyzer as well. The measurements of the electric field inside the waveguide are evaluated in terms of the magnitude and phase of the transmission coefficient $S_{21}$ between the input of the source and output of the receiver antenna. Due to the two-dimensional nature of the parallel plate waveguide, as well as symmetry of our sample, the electric field in the scanned area is expected to remain polarized mainly perpendicular to the plane of the plates.

In order to analyze the power-induced shift of the magnetic resonance due to the action of the varactor diodes introduced into split-ring resonators, we measure the transmission of the magnetic metamaterial for different values of the input power. Figure 2(right) shows the dependence of the transmission coefficient on the frequency at three different values of the input power. Similar to the nonlinearity-induced effects observed for a single split-ring resonator,, 910 the resonant frequency is shifted to the right when the input power grows. These results show that, by selecting the operational frequency near the resonance, we may change dynamically the transmission properties of the metamaterial by varying the input power. 

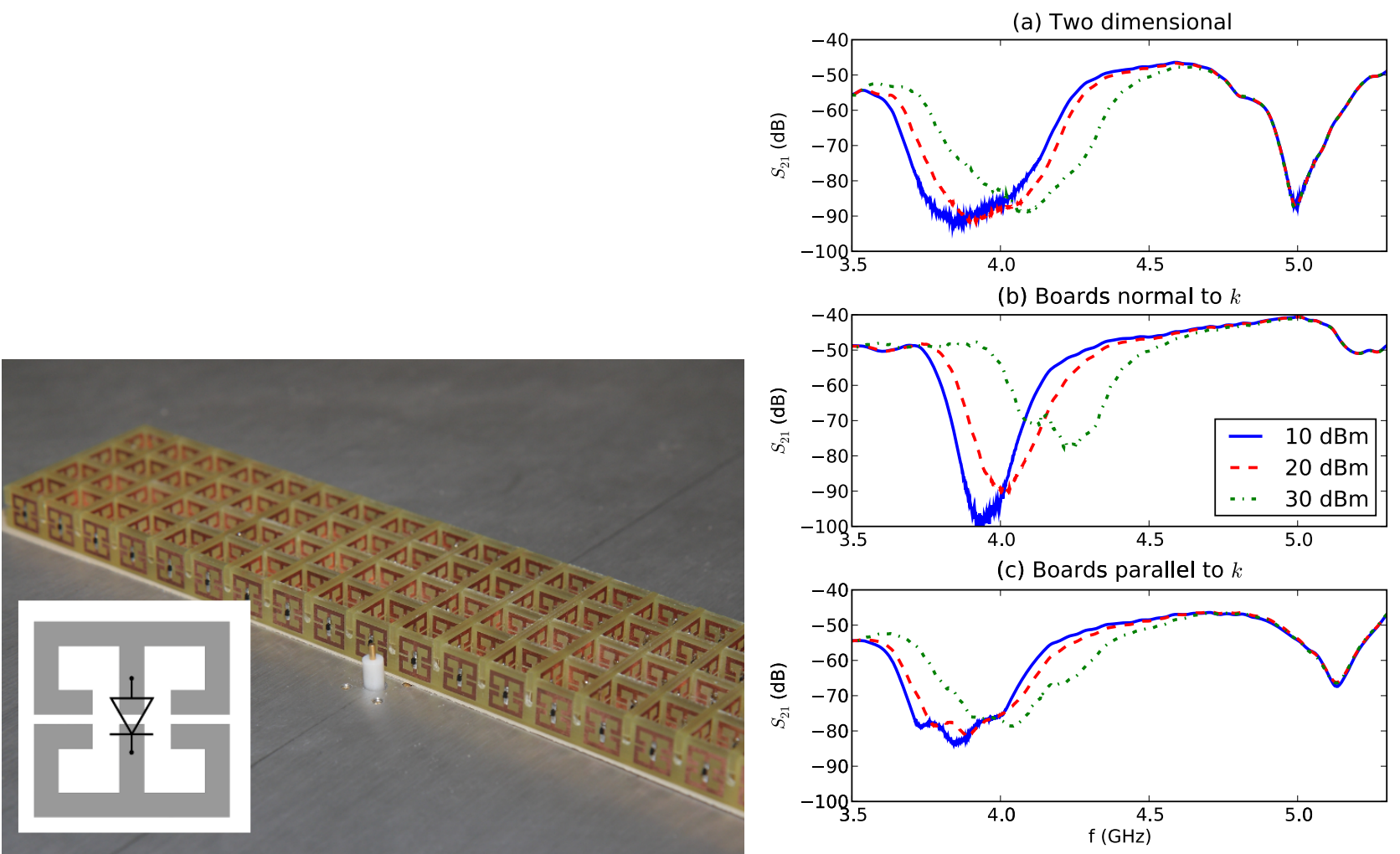

(b) Boards normal to $k$

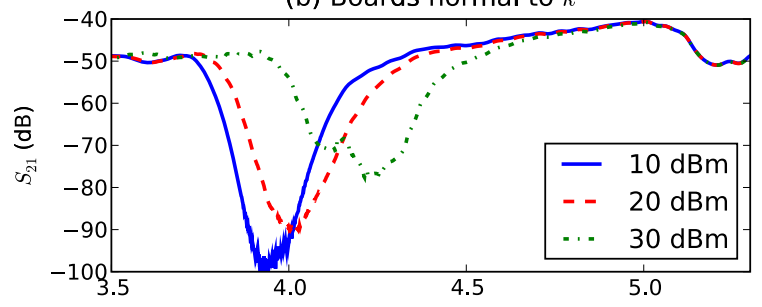

(c) Boards parallel to $k$

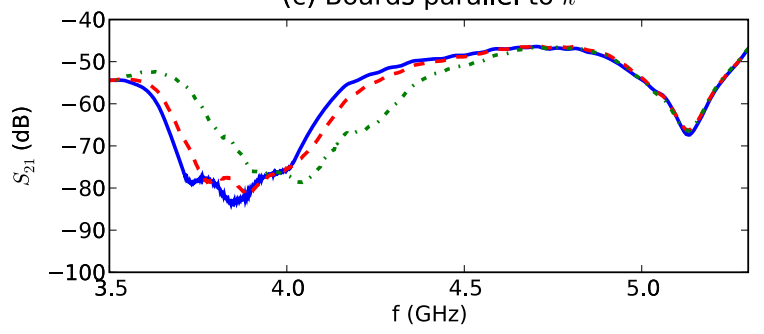

Figure 3. Left: Slab of nonlinear electric metamaterials used in our experiments. The inset shows one structure element. Right: (a-c) Experimental nonlinear transmission response at incident powers of 10, 20 and $30 \mathrm{dBm}$ for different orientations.

If the parameters of a metamaterial vary and cross the boundary between positive and negative values of the effective magnetic permeability, the structure properties such as transmission will be switched as well, so that the material itself will change from opaque to transparent. The intensity of electromagnetic waves generated by a point source is non-uniform, so that a shift of resonances of individual split-ring resonators is inhomogeneous inside the metamaterial structure. The resonators closer to the source will experience stronger fields and thus it is expected that only the central part of the metamaterial becomes transparent. The experimental results confirm this effect by revealing a narrow aperture of the beam emerging from the metamaterial. ${ }^{22}$ In the same metamaterial sample we can observe the opposite effect when at the high-frequency side of the resonance the transmission is suppressed by the nonlinearity. While the metamaterial is transparent for low powers, the growth of the wave amplitude makes a part of the metamaterial opaque, and it prevents the radiation to go through the sample.

\section{NONLINEAR ELECTRIC METAMATERIALS}

Given that the nonlinear shift of the resonance results in a very strong nonlinear magnetic response for split-ring resonators, recently we took a similar approach to the design of nonlinear electric resonators, in order to obtain a strong nonlinear electric response. ${ }^{23}$ Our structure is shown in Fig. 3(left), where two perpendicular sets of boards are introduced to create a relatively isotropic response. Within each resonator, an additional gap is introduced where a varactor diode is placed, introducing additional series capacitance in order to tune the resonant frequency. The lattice period is $11 \mathrm{~mm}$, and the resonators are fabricated on copper-clad FR4 with width and height of $8 \mathrm{~mm}$, track width of $1 \mathrm{~mm}$, with outer gaps of $0.4 \mathrm{~mm}$ separation and $2.4 \mathrm{~mm}$ length. 
We measure the transmission response at incident powers of 10, 20 and 30dBm as shown in Fig. 3(right). At the lowest incident power $(10 \mathrm{dBm})$, there is negligible tuning of the response by the incident wave, thus the transmission response in this case is essentially linear. The large insertion loss away from resonance is due to the deliberately mismatched receiving probe which is designed for minimal perturbation of the fields within the structure.

Figure 3(right, a) shows that the higher frequency mode does not shift its frequency with a change of the incident power. This mode consists of two current loops flowing in the same direction, thus their magnetic dipole moments add constructively. As the accumulated charges across the gaps have opposite directions, this results in a vanishing electric dipole moment. As there is no net current through the central conductor, the nonlinear response of the varactor diode does not come into play. These mode configurations, and the stop-band locations, are confirmed by numerical simulations of transmission performed in CST Microwave Studio using a single element with electric boundaries in the vertical direction and magnetic boundaries in the horizontal direction.

For comparison, we investigate the two different orientations of the circuit boards individually, i.e. those which are normal to the direction of propagation, and those which are parallel to it. The nonlinear transmission responses for these structures are shown in Fig. 3(right, b) and Fig. 3(right, c), respectively. In both cases a significant nonlinear response still occurs. In the case of the boards being perpendicular to the direction of propagation, the higher frequency magnetic stop-band does not exist. This is due to the symmetry of the fields across the gaps and the lack of any magnetic field component normal to the rings. We note that as the experiment uses a cylindrically symmetric source there is some component of the wavenumber normal to the nominal propagation direction, hence some vestige of the second resonance remains. Also of note is the fact that both resonances are noticeably modified in the isotropic configuration compared to when they are measured separately. This is likely to be due to the strong electrical interaction between the nearest-neighbor boards in the orthogonal directions, due to their gaps being in close proximity.

\section{STRUCTURAL TUNABILITY OF METAMATERIALS}

Finally, in this last section we discuss and demonstrate experimentally a novel approach for efficient control of the transmission characteristics of metamaterials based on the structural tunability. This concept is rather general, and it is applicable to various metamaterials as long as the effective medium description is valid.

We consider an anisotropic metamaterial based on split-ring resonators, as shown in Fig. 4(left). For sufficiently dense arrays, the interaction between the elements differs considerably from a dipole approximation, and the specific procedure to calculate the effective permeability has been developed earlier ${ }^{24}$; the latter converges correctly to a Clausius-Mossotti approximation in the limit of sparse lattice. Consequently, the effect of mutual coupling is enhanced dramatically as compared to conventional materials, and therefore it is particularly suitable to demonstrate the efficiency of lattice tuning.

The most straightforward lattice tuning approach is to vary the lattice constant $b$. It was shown ${ }^{24}$ that the resonance frequency can be remarkably shifted this way, and this prediction was confirmed by microwave experiments. ${ }^{16}$ Accordingly, a slab of metamaterial can be tuned between transmission, absorption and reflection back to transmission. A clear disadvantage of this method is that varying $b$ significantly would imply a corresponding change in the overall dimension of metamaterial along $z$, which might be undesirable for certain applications.

Following the recent original paper, ${ }^{17}$ here we discuss the basic principles of the structural tuning of the structure by changing a periodic lateral displacement of layers in $x y$ plane, so that the resonators become displaced along $x$ ( $y$, or both) by a fraction of the lattice constant $\delta a$ per each $b$ distance from a reference layer with respect to original position. This decreases the overall mutual inductance in the system and leads to a remarkable gradual increase in resonant frequency, with a maximal effect archived for a displacement by $0.5 a$. Clearly, further shift is equivalent to smaller shift values until the lattice exactly reproduces itself for the shift equal $a$. As a consequence, resonance of the medium can be "moved" across a signal frequency, leading to drastic change in transmission characteristics. It is clear that for practical applications it is not even necessary to exploit the whole range of lateral shift — in the above example it is sufficient to operate between $0.1 a$ and $0.3 a$ where most of the transition occurs. 

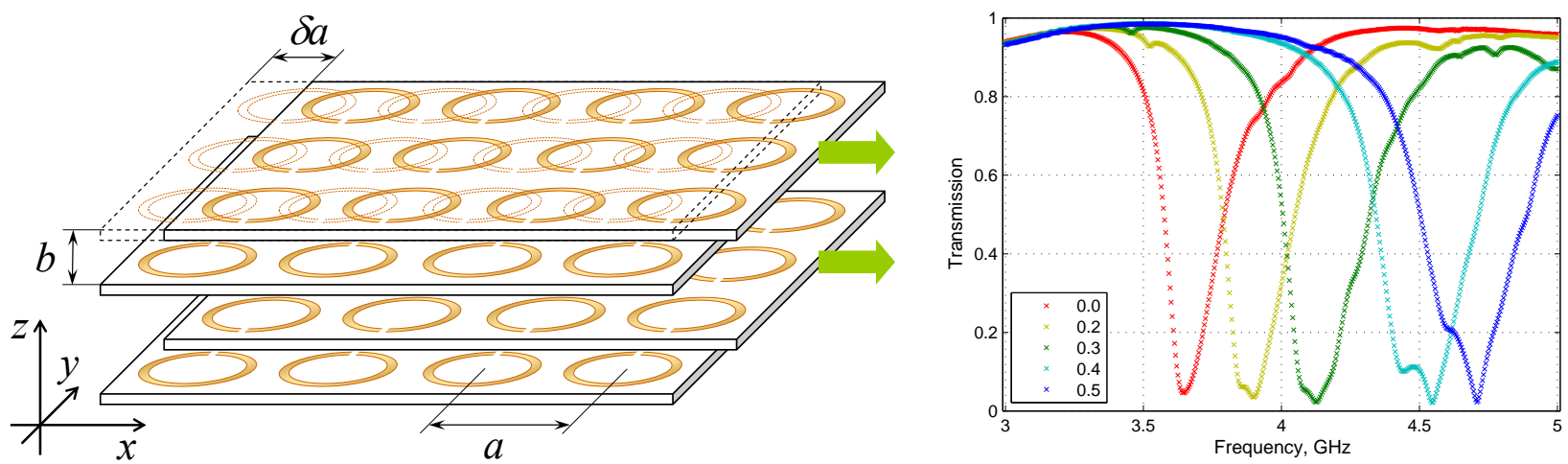

Figure 4. Left: Schematic of one of the realizations of the lattice tuning concept in metamaterials. Right: Experimental transmission in a waveguide with metamaterial slab at different shifts. Broadside-coupled orientation of layers. Curves with dips from left to right correspond to increasing lattice shift.

For the proof of concept, we opted for a small reconfigurable system, built up of single-split rings $(2.25 \mathrm{~mm}$ mean radius, $0.5 \mathrm{~mm}$ strip width, $1 \mathrm{~mm}$ gap) printed with a period $a=7 \mathrm{~mm}$ on $1.5 \mathrm{~mm}$ thick circuit boards. We have 5 resonators in propagation direction $x$ and only one period along $y$; 30 boards are stacked together in $z$ direction with minimal possible lattice constant $b=1.5 \mathrm{~mm}$ used for the measurements. Estimated resonance frequency of a single resonator (in a dielectric environment) is about $4.9 \mathrm{GHz}$, however the resonance of the dense metamaterial is significantly shifted to lower frequencies. To minimize undesirable bianisotropic effects bound to single-split rings, the boards are assembled so that the gaps are oppositely oriented in adjacent layers [see Fig. 4(left)], resembling the logic of broadside-coupled split-ring resonators. ${ }^{25}$ Transmission measurements (Rohde and Schwarz ZVB network analyzer) were performed for various lattice shifts in WR-229 rectangular waveguide.

The experimental transmission spectra are shown in Fig. 4(right), and they demonstrate excellent tuning of the resonance frequency. Furthermore, comparison of the experimental resonance shift with the theoretical predictions shows ${ }^{17}$ that the experimental system demonstrates even higher efficiency. This effect can be explained by accounting for mutual capacitance between the resonators, which is neglected in our theoretical calculations. Indeed, for the broadside-like configuration of rings, mutual capacitance between them is distributed along the whole circumference. ${ }^{25}$ Clearly, when the resonators are laterally displaced, the mutual capacitance decreases, so that this effect is added up to the increase of resonance frequency imposed by decreased inductive coupling.

\section{CONCLUSIONS}

We have presented a brief overview of the recent experimental results from our Nonlinear Physics Center on a design, manufacturing, and characterization of tunable and nonlinear metamaterials operating at microwave frequencies. Such structures can exhibit either nonlinear magnetic or nonlinear electric response at microwave frequencies, and they are created by introducing a varactor diode as an externally tunable and nonlinear element within each resonator of the structure. By engineering a resonance of the split-ring resonator, we are able to modify the averaged response of the composite structure making its parameters dependent on both the external bias and incident power. We also discuss a recently suggested and demonstrated novel approach for an efficient tuning of the transmission characteristics of metamaterials through a continuous adjustment of the lattice structure, the structural tunability. Some of the tuning mechanisms discussed here can be suitable for scaling toward optical wavelengths.

\section{Acknowledgements}

We would like to thank the members and visitors of our Nonlinear Physics Center, as well as our collaborators who co-authored the original results summarized in this conference paper, including Maxim V. Gorkunov, Alexander 
B. Kozyrev, Michael Lapine, Steven K. Morrison, David A. Powell, and Ilya V. Shadrivov. This work was supported by the Australian Research Council.

\section{REFERENCES}

1. J.B. Pendry, A.J. Holden, W.J. Stewart, and I. Youngs, Phys. Rev. Lett. 76, 4773 (1996).

2. J.B. Pendry, A.J. Holden, D.J. Robbins, and W.J. Stewart, IEEE Trans. Microwave Theory Tech. 47, 2075 (1999).

3. P. Markos and C.M. Soukoulis, Phys. Rev. E 65, 036622(2002); Phys. Rev. B 65, 033401 (2002).

4. D.R. Smith, W. Padilla, D.C. Vier, S.C. Nemat-Nasser, and S. Shultz, Phys. Rev. Lett. 84, 4184 (2000).

5. C.G. Parazzoli, R.B. Greegor, K. Li, B.E.C. Koltenbah, and M. Tanielian, Phys. Rev. Lett. 90, 107401 (2003).

6. V. G. Veselago, Sov. Phys. Uspekhi 8, 2854 (1967) [Sov. Phys. Usp. 10, 509 (1968)].

7. M. Lapine and S. Tretykov, IET Microwaves Antennas \& Propagation 1, 3 (2007).

8. A. Sihvola, Metamaterials 1, 2 (2007).

9. I.V. Shadrivov, S.K. Morrison, and Yu.S. Kivshar, Optics Express 14, 9344 (2006).

10. D.A. Powell, I.V. Shadrivov, and Yu.S. Kivshar, and M.V. Gorkunov, Appl. Phys. Lett. 91, 144107 (2007).

11. B. Wang, J. Zhou, T. Koschy, and C.M. Soukoulis, Opt. Express 16, 16058 (2008).

12. K.A. Boulais, D.W. Rule, S. Simmons, F. Santiago, V. Gehman, K. Long, and A. Rayms-Keller, Appl. Phys. Lett. 93, 043518 (2008).

13. S. O’Brien, D. McPeake, S.A. Ramakrishna, and J.B. Pendry, Phys. Rev. B 69, 241101 (2004).

14. Z. Sheng and V.V. Varadan, J. Appl. Phys. 101, 014909 (2007).

15. N.-H. Shen, M. Kafesaki, T. Koschny, L. Zhang, E.N. Economou, and C. Soukoulis, Phys. Rev. B 79, 161102(R) (2009).

16. I.V. Shadrivov, D.A. Powell, S.K. Morrison, Yu.S. Kivshar, and G.N. Milford, Appl. Phys. Lett. 90, 201919 (2007).

17. M. Lapine, D. Powell, M. Gorkunov, I. Shadrivov, R. Marqués, and Yu.S. Kivshar, "Structural tunability in metamaterials", submitted to Appl. Phys. Lett (2009).

18. A.A. Zharov, I.V. Shadrivov, and Yu.S. Kivshar, Phys. Rev. Lett. 91, 037401 (2003).

19. M. Lapine, M. Gorkunov, and K.H. Ringhofer, Phys. Rev. E 67, 065601 (2003).

20. M. Gorkunov and M. Lapine, Phys. Rev. B 70, 235109 (2004).

21. I.V. Shadrivov, A.B. Kozyrev, D.W. van der Weide, and Yu.S. Kivshar, Appl. Phys. Lett. 93, 161903 (2008).

22. I.V. Shadrivov, A.B. Kozyrev, D.W. van der Weide, and Yu.S. Kivshar, Opt. Express 16, 20266 (2008).

23. D.A. Powell, I.V. Shadrivov, and Yu.S. Kivshar, "Nonlinear electric metamaterials", submitted to Appl. Phys. Lett (2009).

24. M. Gorkunov, M. Lapine, E. Shamonina, and K. H. Ringhofer, Eur. Phys. J. B 28, 263 (2002).

25. R. Marqués, F. Mesa, J. Martel, and F. Medina, IEEE Trans. Anten. Propag. 51, 2572 (2003). 